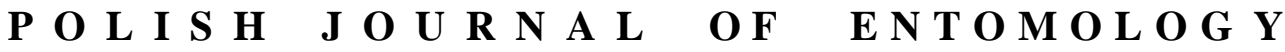

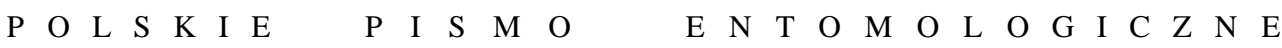

VOL. 87: 153-164

Lublin

30 June 2018

DOI: $10.2478 /$ pjen-2018-0011

\section{New records for fauna of the subfamily Dexiinae (Diptera: Tachinidae) in Iran}

\author{
FARNAZ SEYYedi SAHEBARI ${ }^{1,2^{*}}$, SAMAD KHAGHANINIA ${ }^{1}$, \\ ALI ASGHAR TALEBI ${ }^{3}$ \\ ${ }^{1}$ Department of Plant Protection, Faculty of Agriculture, University of Tabriz, 51664, \\ Tabriz, Iran \\ ${ }^{2}$ Plant Protection Research Department, East Azarbaijan Agricultural and Natural \\ Resources Research and Education Center, AREEO, Tabriz, Iran \\ ${ }^{3}$ Department of Entomology, Faculty of Agriculture, Tarbiat Modares University, \\ 14115-336, Tehran, Iran
}

\begin{abstract}
This study was carried out to identify the Tachinidae fauna in northern Iran during 2010-2011 and ten species belonging to nine genera and two tribes of the subfamily Dexiinae were found. The species Estheria petiolata, Periscepsia spathulata, Stomina caliendrata and Thelaira nigripes are new records for the Iranian tachinid fauna.
\end{abstract}

KEY WORDS: Diptera, Tachinidae, Dexiinae, Parasitoid, Iran, new records.

\section{INTRODUCTION}

Tachinidae is one of the most diverse dipteran families, with more than 8500 species worldwide (O'HARA 2013). Nearly all the members of this family are entomophagous parasitoids and have an important role as biocontrol agents of pests in agricultural and forest ecosystems. Many tachinids have been successfully used in biological control programmes (GRENIER 1988, STIREMAN et al. 2006). The Tachinidae are divided into four subfamilies: Phasiinae, Dexiinae, Exoristinae and Tachininae. The Dexiinae are the only subfamily widely regarded as monophyletic, a belief based on shared derived features of

\footnotetext{
*Corresponding author: f_seyyedi_sahebari@yahoo.com
} 
the male terminalia. In particular, male dexiines possess a hinged connection between the basiphallus and distiphallus that is not found in other tachinids (TsCHORSNIG 1985, WOOD 1987). This subfamily has about 54 genera and 236 species in the Palearctic region (HERTING \& DELY-Draskovits 1993, O'HARA 2013) and the species of this subfamily commonly parasitize larval Coleoptera (mostly Scarabaeidae) and Lepidoptera.

The history of studies on Tachinidae fauna in Iran is not very long and only a few short notes have been published about Iranian Dexiinae. In the first study, SAMET et al. (1977) reported the species Billaea biserialis (PORTSHINSKY, 1881), Billaea zimini KOLOMIETS, 1966 and Periscepsia carbonaria (PANZER, 1798) in the "Preliminary list of Diptera of Iran". Later, HeRTING (1984) added Estheria pallicornis (LOEW, 1873) and PARCHAMI ARAGHI (1995) reported the species Voria ruralis (FALLÉN, 1810) as a parasitoid of larval Autographa gamma (LinNAEUs, 1758). GHEIBI et al. $(2008,2009)$ reported four species Athrycia impressa (VAN DER WULP, 1869), Cyrtophloeba ruricola (MEIGEN, 1824), Periscepsia carbonaria (PANZER, 1798) and Thelaira solivaga (HARRIS, 1780) - from Fars province. ZIEGLER et al. (2016) described the subspecies of Dinera fuscata occidentalis ZIEGLER, 2013 from Mazandaran and East Azerbaijan provinces. Prior to this study, we reported 17 tachinid species belonging to 14 genera of the subfamily Dexiinae from northwestern Iran, of which 14 species and ten genera were new records for the Tachinidae fauna of Iran (SEYYEDI SAHEBARI et al. 2014).

This study aims to extend the checklist of Iranian tachinid species and to add further data on their distributions in Iran.

\section{MATERIALS AND METHODS}

Twelve Malaise traps were deployed in grasslands and forests of Gilan and Mazandaran provinces, situated in northern Iran. The forests are named after the ancient region of Hyrcania. This region includes the coast along the Caspian Sea and the northern slopes of the Alborz Mountains (Fig. 1). The climate in this region is humid subtropical maritime in middle altitude locations, while in the mountains it is humid continental with most precipitation occurring in autumn, winter and spring. The specimens were collected from April to October in 2010 and 2011. They were deposited at the Faculty of Agriculture, Tarbiat Modares University, Tehran, Iran, and in the collection of Professor Hasan MALEKI MILANI, Tabriz University, Iran (ICHMM). The specimens were identified on the basis of the keys by MESNIL (1944-1975, 1980), TSCHORSNIG \& HERTING (1994) and TsCHORSNIG \& RICHTER (1998). The photographs were taken using a Nikon SMZ 1000 stereomicroscope equipped with a Nikon D5200 digital camera. 


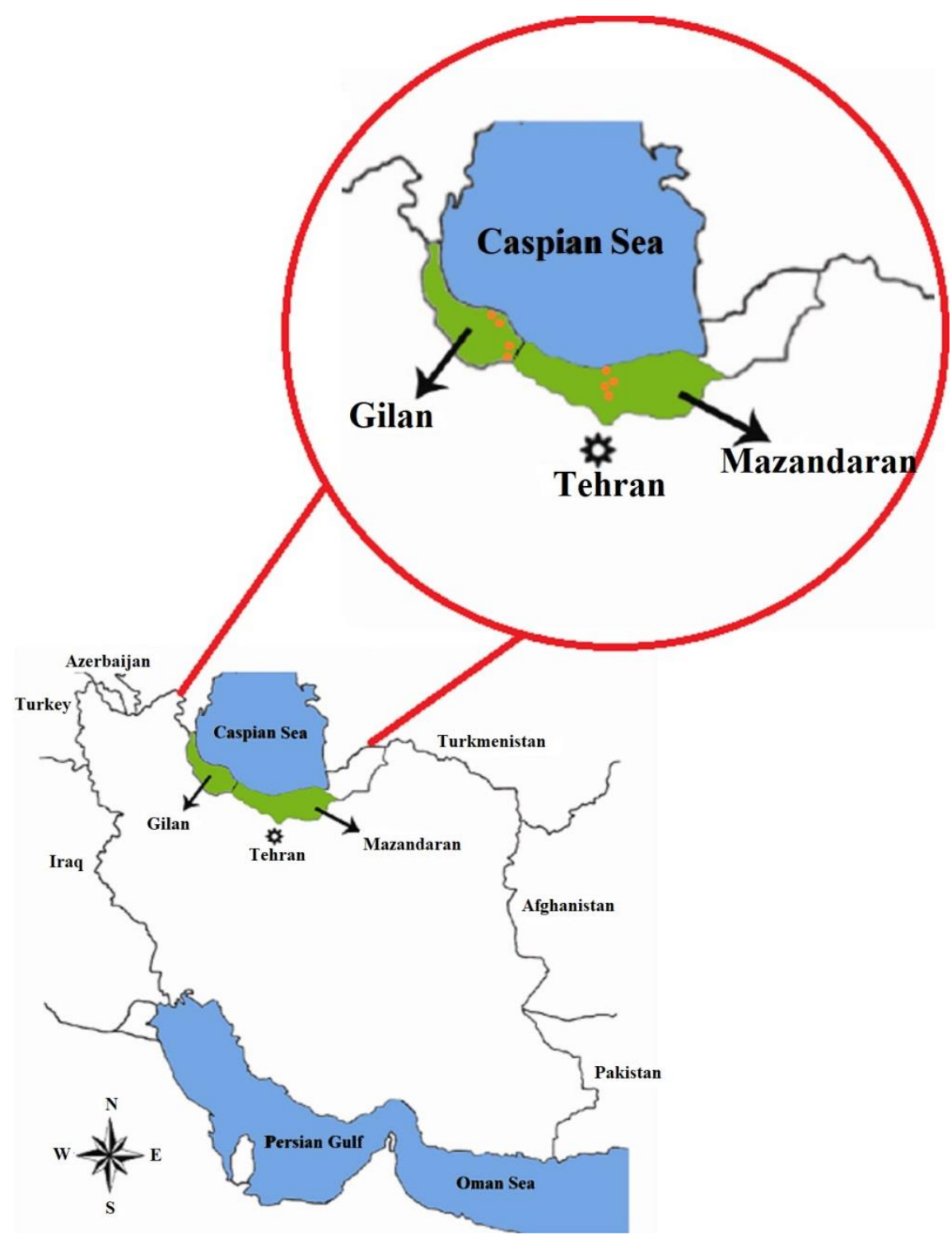

Fig. 1. Gilan and Mazandaran provinces, where the material for this study was collected.

\section{List of localities}

Gilan province:

Roodsar, Eshman Kamachal, 37²2’3.66”N, 4957'57.84’E, 1 m, 37²1'10.50”N, $49^{\circ} 57^{\prime} 56.16^{\prime \prime} \mathrm{E}, 2 \mathrm{~m}$; vegetation: rice field, area near the sea. 
Roodsar, Ghazichak, $\quad 36^{\circ} 45^{\prime} 5.54^{\prime \prime} \mathrm{N}, \quad 50^{\circ} 19^{\prime} 35.22^{\prime \prime} \mathrm{E}, \quad 1803 \mathrm{~m}, \quad 36^{\circ} 45^{\prime} 52.62^{\prime \prime} \mathrm{N}$, $50^{\circ} 20^{\prime} 1.08^{\prime \prime} \mathrm{E}, 1787 \mathrm{~m}$; vegetation: grassland, barberry bush.

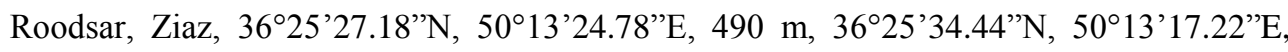
$537 \mathrm{~m}$; vegetation: hazelnut garden.

Roodsar, Orkom, $36^{\circ} 45^{\prime} 44.344^{\prime} \mathrm{N}, 50^{\circ} 18^{\prime} 11.88^{\prime \prime} \mathrm{E}, 1201 \mathrm{~m}$; vegetation: mixed deciduous forest, hazelnut garden.

Mazandaran province:

Ghaznasara, 36 $16^{\prime} 56.82^{\prime \prime} \mathrm{N}, 52^{\circ} 10^{\prime} 58.50^{\prime \prime} \mathrm{E}, 2032 \mathrm{~m}, 36^{\circ} 16^{\prime} 58.08^{\prime \prime} \mathrm{N}, 52^{\circ} 10^{\prime} 55.62^{\prime \prime} \mathrm{E}$, $2013 \mathrm{~m}$; vegetation: grassland, shrub.

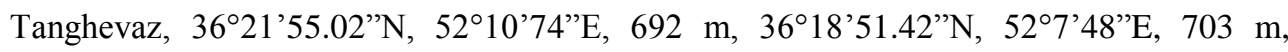
$36^{\circ} 21^{\prime} 55.68^{\prime \prime} \mathrm{N}, 52^{\circ} 6^{\prime} 10.32^{\prime \prime} \mathrm{E}, 702 \mathrm{~m}$; vegetation: deciduous and coniferous forest.

\section{RESULTS}

In the course of the study, 52 individuals of the subfamily Dexiinae were collected and identified; they belong to ten species, nine genera and two tribes. Four species are new records (marked with an asterisk) for the Iranian Tachinidae fauna. The diagnostic characters of the first recorded species are documented with photographs. All altitudes are given in metres above mean sea level (m).

\section{Tribe: Dexiini \\ Genus: Dexia MeIGEN, 1826}

\section{Dexia rustica (FABRICIUS, 1775)}

Material examined: $\left(1 \delta^{\Uparrow}\right), 20 . V .2010 \&\left(1 \delta^{\Uparrow}\right)$, 18.VI.2010, Gilan province, Roodsar

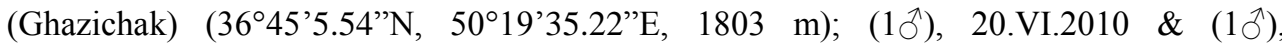

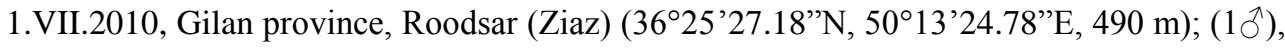

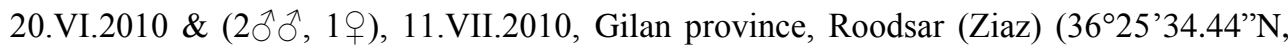
$50^{\circ} 13^{\prime} 17.22^{\prime \prime} \mathrm{E}, 537 \mathrm{~m}$ ), leg. M. KHEIRANDISH; (1ㅇ), Mazandaran province, Ghaznasara $\left(36^{\circ} 16^{\prime} 56.82^{\prime} \mathrm{N}, 52^{\circ} 10^{\prime} 58.50^{\prime} \mathrm{E}, 2032 \mathrm{~m}\right)$, 4.VII.2011; (1 $\left.{ }^{\Uparrow}\right)$, Mazandaran province,

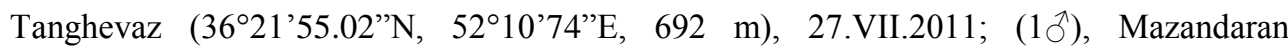
province, Tanghevaz ( $36^{\circ} 18^{\prime} 51.42^{\prime}$ 'N, 52 $2^{\circ} 7^{\prime} 48^{\prime \prime}$ E, $\left.703 \mathrm{~m}\right)$, 4.VIII.2011, leg. A. NADIMI.

Distribution: All Europe (TSCHORSNIG et al. 2017). Asia: Transcaucasia, southern and western Siberia (HERTING 1984). Iran (SEYYEDI SAHEBARI et al. 2014).

Host range: Melolontha sp.; Amphimallon solstitialis (LinNAEUs, 1758); Phyllopertha horticola (LINNAEUS, 1758); Rhizotrogus aequinoctialis (HERBST, 1790); R. marginipes MULSANT, 1842 (Coleoptera: Scarabaeidae) (TSCHORSNIG \& HERTING 1994).

Genus Estheria ROBINEAU-DESVOIDY, 1830 


\section{Estheria petiolata (BONSDORFF, 1866) * (Fig. 2)}

Material examined: $\left(1 \delta^{\Uparrow}\right)$, Mazandaran province, Ghaznasara $\left(36^{\circ} 16^{\prime} 56.82^{\prime} \mathrm{N}\right.$, 52॰10'58.50’'E, $2032 \mathrm{~m})$, 27.VII.2011; (1今), Mazandaran province, Tanghevaz (36²1'55.68''N, 526'10.32”E, 702 m), 4. VIII. 2011, leg. A. NADIMI.

Remarks: European and Mediterranean species of male Estheria have a darker scutellum near the anterior margin and partly yellow mid-tibiae (CERLETTI \& TSCHORSNIG 2012). The scutellum and legs of our male specimens are entirely black. We take these to be intraspecific variations.

Distribution: Europe: Finland, Spain, France, Belarus, Ukraine (TsCHORSNIG et al. 2017). Asia: Transcaucasia, Kazakhstan and Central Asia (Kyrgyzstan, Tajikistan, Turkmenistan, Uzbekistan) (HERTING \& DELY-DRASKOVITS 1993). New record for Iran.

Host range: Amphimallon solstitialis (Coleoptera: Scarabaeidae) (TSCHORSNIG \& HERTING 1994).
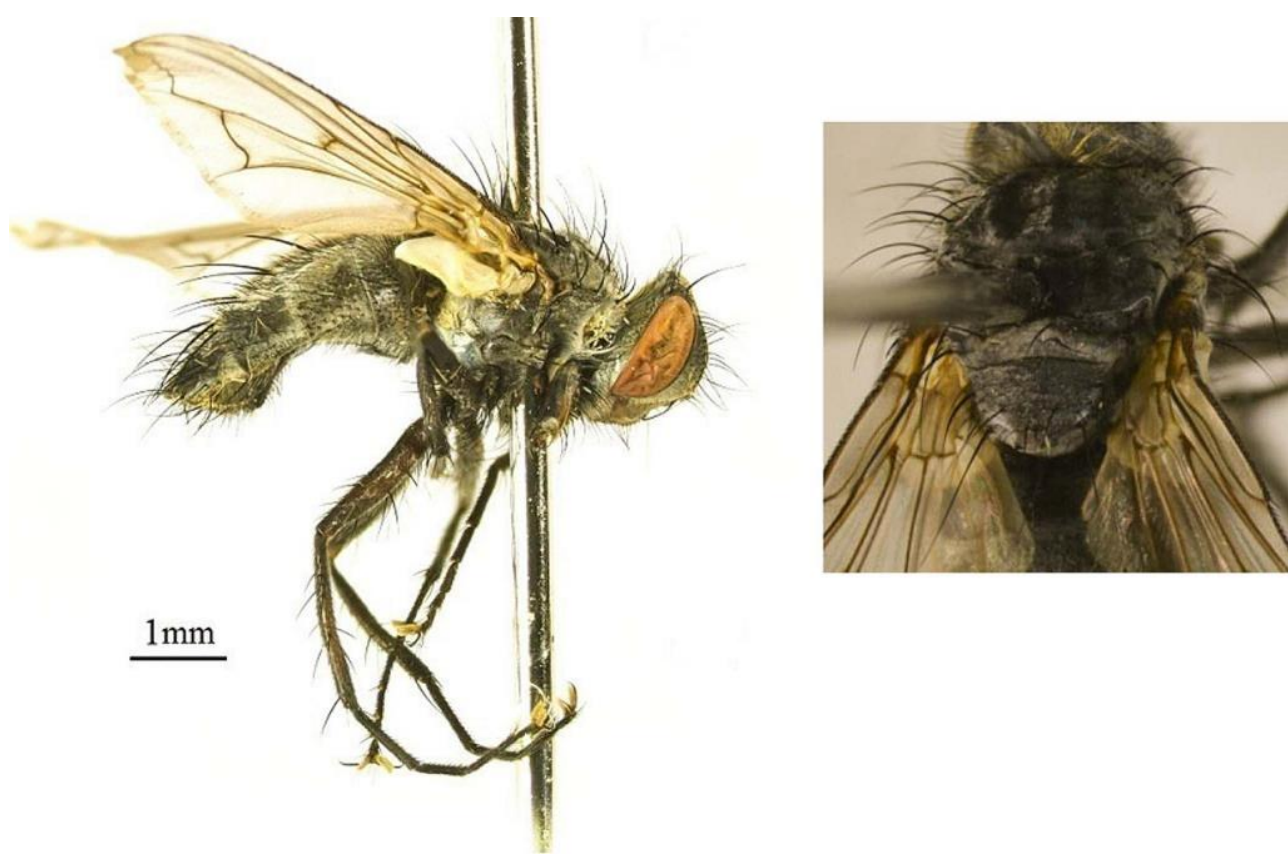

Fig. 2. Estheria petiolata (BONSDORFF, 1866), male. 


\section{Tribe: Voriini}

\section{Genus Athrycia ROBINEAU-DESVOIDY, 1830}

Athrycia trepida (MEIGEN, 1824)

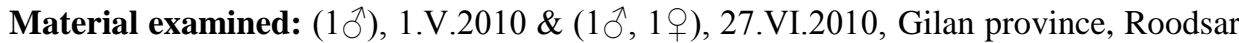
(Orkom) (3645'44.34’N, 50¹8'11.88’'E, $1201 \mathrm{~m})$, leg. M. KHEIRANDISH.

Distribution: Europe: Sweden, Finland, Italy, Greece, Bulgaria, Spain, France, Belarus, Ukraine (TsCHORSNIG et al. 2017) Asia: Transcaucasia, Central Asia, Middle East, Mongolia, Russia (Herting \& Dely-Draskovits 1993). Iran (SEYYedi SAHEBARI et al. 2014).

Host range: Orthosia gothica (LiNNAEUS, 1758) (Lepidoptera: Noctuidae) (TSCHORSNIG \& HERTING 1994).

\section{Genus Cyrtophleba Rondani, 1856}

\section{Cyrtophleba ruricola (MEIGEN, 1824)}

Material examined: (1 $\left.\delta^{\Uparrow}\right)$, Gilan province, Roodsar (Orkom) (36 $45^{\prime} 44.34^{\prime \prime} \mathrm{N}$, 50¹8’11.88’E, 1201 m), 27.VI. 2010, leg. M. KHEIRANDISH.

Distribution: Europe: Widespread in Europe (TSCHORSNIG et al. 2017). Asia: Transcaucasia, Central Asia, Mongolia, Israel, south-eastern Siberia (HERTING \& DELYDRASKOVITS 1993). Iran (GHEIBI et al. 2008).

Host range: Apopestes spectrum (ESPER, 1787) (Lepidoptera: Noctuidae); Deilephila elpenor (LINNAEUS, 1758) (Lepidoptera: Sphingidae); Pachycnemia hippocastanaria (HÜBNER, 1799) (Lepidoptera: Geometridae) (TSCHORSNIG \& HERTING 1994).

\section{Genus Eriothrix MEIGEN, 1803}

\section{Eriothrix rufomaculatus (DE GEER, 1776)}

Material examined: (1ठ), 1.V.2010 \& (1ठ), 1.X.2010, Gilan province, Roodsar

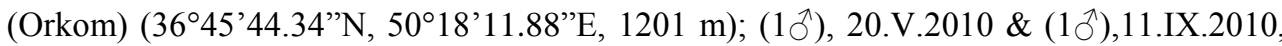
Gilan province, Roodsar (Ghazichak) $\left(36^{\circ} 45^{\prime} 52.62^{\prime}\right.$ N, 50²0’1.08”E, $\left.1787 \mathrm{~m}\right)$, leg. M. KHEIRANDISH $\left(1 \circlearrowleft^{\Uparrow}\right), 4 . V I .2010$ \& $\left(1 \circlearrowleft^{\Uparrow}\right)$, 27.IX.2010, Mazandaran province, Ghaznasara $\left(36^{\circ} 16^{\prime} 56.82^{\prime} \mathrm{N}, 52^{\circ} 10^{\prime} 58.50^{\prime \prime} \mathrm{E}, 2032 \mathrm{~m}\right) ;\left(1{ }^{\Uparrow}\right)$, Mazandaran province, Ghaznasara $\left(36^{\circ} 16^{\prime} 58.08^{\prime} \mathrm{N}, 52^{\circ} 10^{\prime} 55.62\right.$ 'E, $\left.2013 \mathrm{~m}\right), 27 . I X .2011$; $\left(1 \bigcirc^{\Uparrow}\right)$, Mazandaran province, Tanghevaz (36²1'55.02”N, 52¹0’74’'E, 692 m), 4.VIII.2011, leg. A. NADIMI.

Distribution: Europe: Sweden, Finland, Norway, Italy, Greece, Bulgaria, Spain, France, Ukraine (TsCHORSNIG et al. 2017). Asia: Middle East, Transcaucasia, Kazakhstan, Uzbekistan, southern Siberia, Russian Far East (ZIEGLER \& SHIMA 1996), Iran (SEYYEDI SAHEBARI et al. 2014). 
Host range: Chrysoteucha culmella (LinNAEUS, 1758) (Lepidoptera: Crambidae) (TSCHORSNIG \& HERTING 1994).

\section{Genus Periscepsia GISTEL, 1848}

\section{Periscepsia carbonaria (PANZER, 1798)}

Material examined: $\left(1{ }^{\AA}\right)$, Gilan province, Roodsar (Ghazichak) $\left(36^{\circ} 45^{\prime} 52.62^{\prime \prime} \mathrm{N}\right.$,

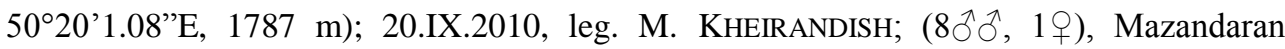

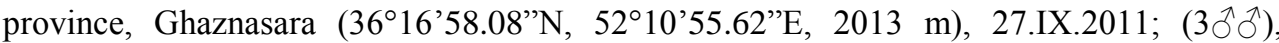
Mazandaran province, Ghaznasara (36¹6’56.82’N, 52¹0’58.50”E, 2032 m), 27.IX.2011, leg. A. NADIMI.

Distribution: Europe: Widespread in Europe (TsChORSNIG et al. 2017). Asia: Transcaucasia, Israel (HERTING \& DELY-Draskovits 1993), Iran (SAMET et al. 1977).

Host range: Agrotis spp., Euxoa obelisca (DENIS et SCHIFFERMÜLleR, 1775) (Lepidoptera Noctuidae) (TsCHORSNIG \& HERTING 1994).

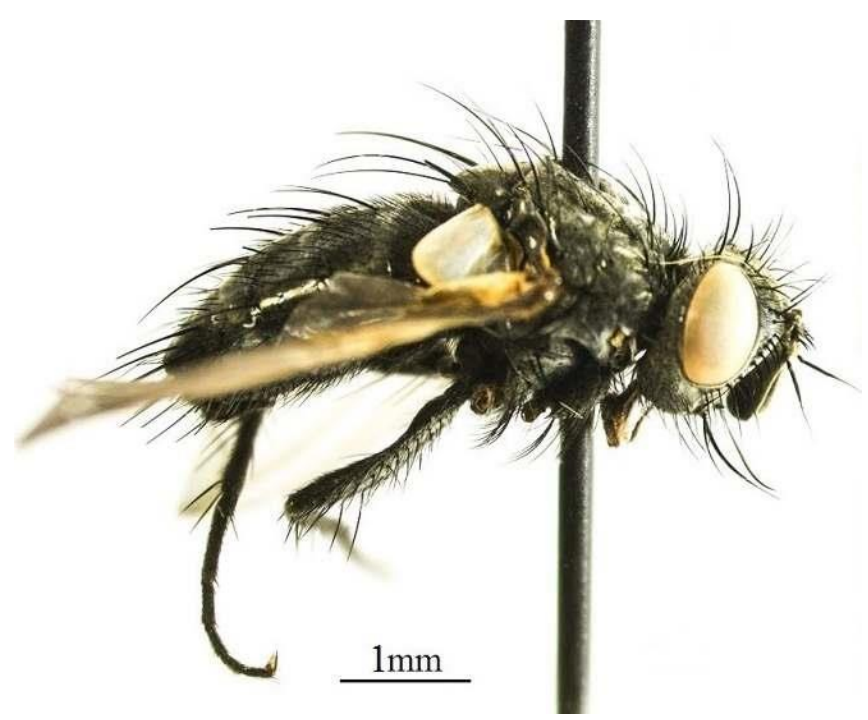

Fig. 3. Periscepsia spathulata (FALLÉN, 1820), male.

\section{Periscepsia spathulata (FALléN, 1820) * (Fig. 3 )}

Material examined: (1 $\left.\delta^{\Uparrow}\right)$, Gilan province, Roodsar (Ziaz) $\left(36^{\circ} 25^{\prime} 34.44^{\prime \prime} \mathrm{N}\right.$, 50¹3'17.22”'E, 537 m); 27.IV.2010; (1 $\left.{ }^{\AA}\right)$, Gilan province, Roodsar (Ziaz) (36 25'27.18’'N, 50¹3'24.78”'E, 490 m), 20.V.2010, leg. M. KHEIRANDISH. 
Distribution: Europe: Sweden, Finland, Norway, Italy, Greece, Bulgaria, Portugal, Spain, France, Russia, Ukraine, Greece, Bulgaria (TsCHORSNIG et al. 2017). Asia: Transcaucasia, China (HERTING \& DELY-DrasKoviTs 1993). New record for Iran.

Host range: Various Noctuidae (Lepidoptera) (TSCHORSNIG \& HERTING 1994).
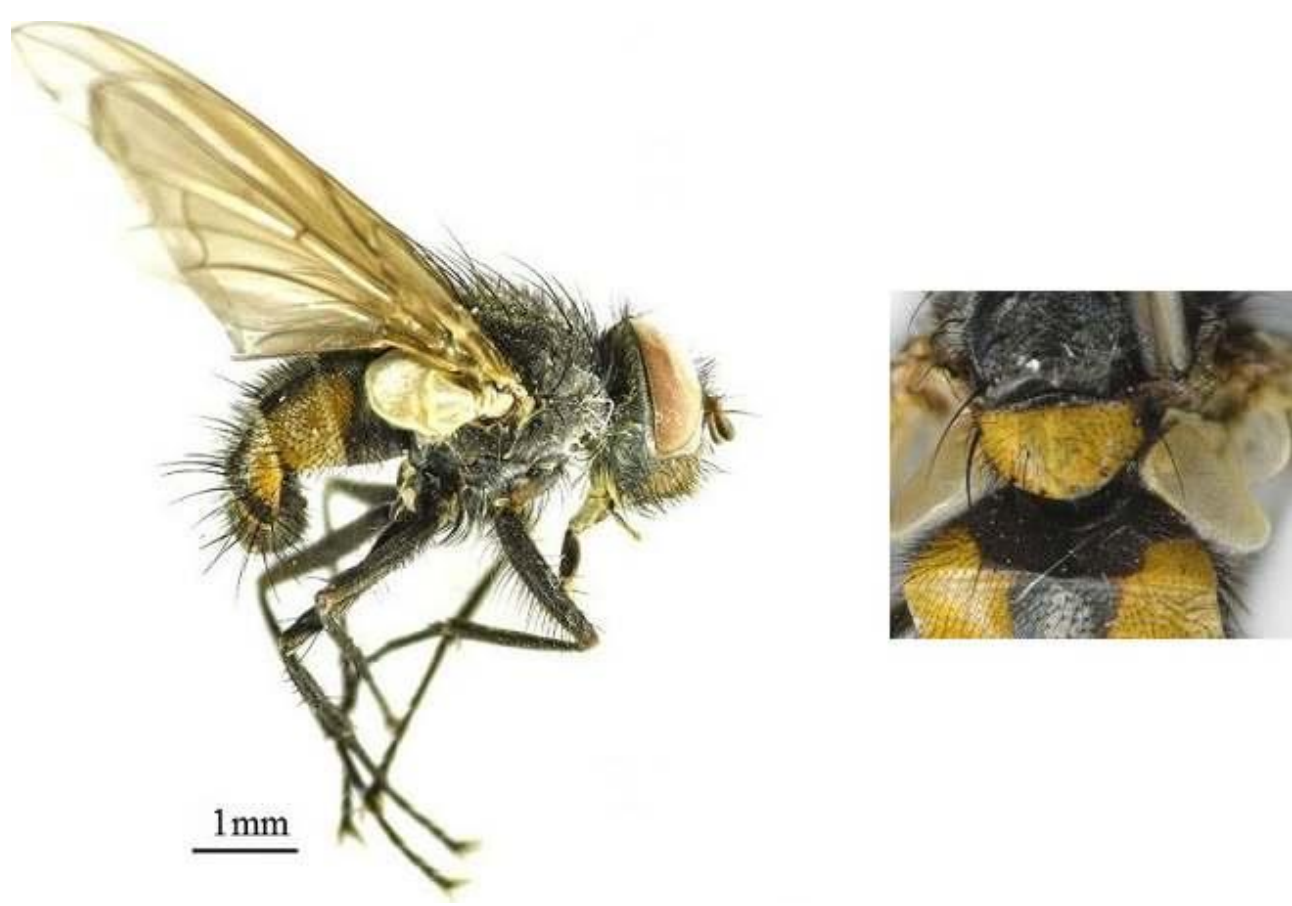

Fig. 4. Stomina caliendrata (RoNDANI, 1862), male.

\section{Genus Stomina ROBINEAU-DESVOIDY, 1830}

Stomina caliendrata $($ RONDANI, 1862) * (Fig. 4)

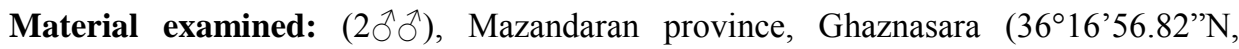
52 $\left.2^{\circ} 10^{\prime} 58.50 ’ \mathrm{\prime}, 2032 \mathrm{~m}\right), 13 . \mathrm{V} .2011$, leg. A. NADIMI.

Distribution: Europe: Portugal, Spain, France, Croatia, Italy, Bulgaria, Ukraine (TsChORSNIG et al. 2017). Asia: Transcaucasia, Israel (HERTING \& DELY-Draskovits 1993). New record for Iran.

Host range: Unknown. 
Remarks: MESNIL (1944-1975: 1817) wrote that the scutellum of S. caliendrata is brown, more or less blackened in its anterior half. Specimens have a yellow scutellum, black on the anterior margin (Fig. 4). We take this to be an intraspecific variation.

\section{Genus Thelaira RoBINEAU-DESvOIDY, 1830}

\section{Thelaira nigripes (FABRICIUS, 1794) * (Fig. 5)}

Material examined: (1ㅇ), Gilan province, Roodsar (Orkom) (36 45'44.34”N,

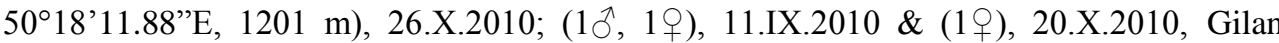
province, Roodsar (Eshman Kamachal) (37²2’3.66”N, 4957’57.84’E, $1 \mathrm{~m}$ ), leg. M. KHEIRANDISH.

Distribution: Europe: Widespread in Europe (TSCHORSNIG et al. 2017). Asia: Transcaucasia, eastern Siberia (HERTING \& DELY-DRASKOVITS 1993). New record for Iran.

Host range: Mainly Arctiidae (Erebidae), but also a few Noctuidae (Lepidoptera) and other Macrolepidoptera (TSCHORSNIG \& HERTING 1994).

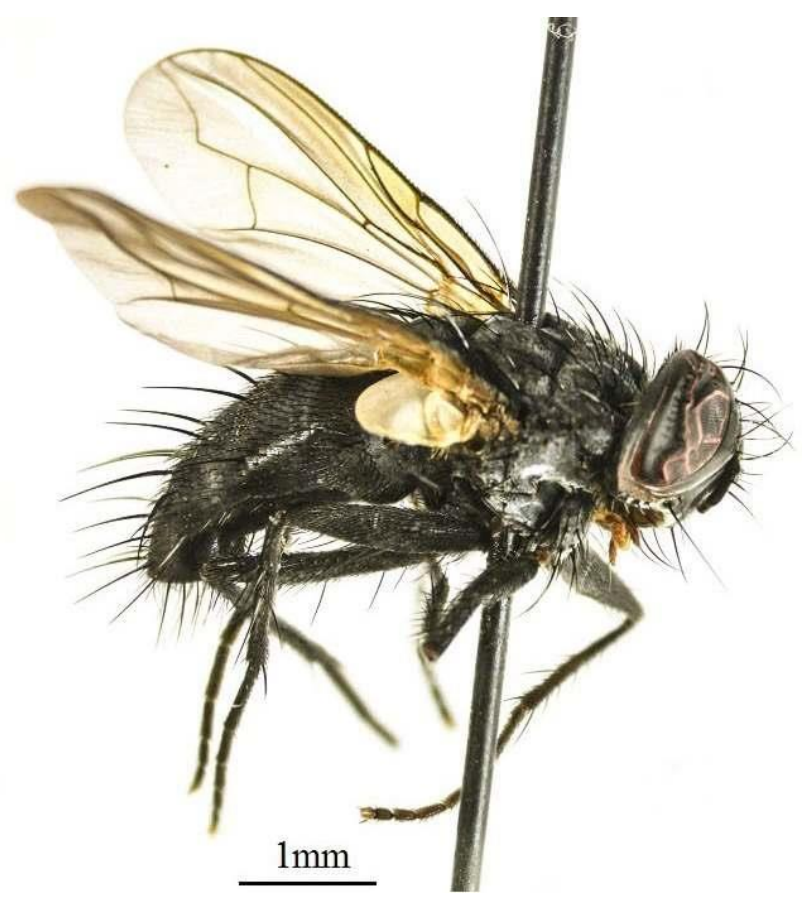

Fig. 5. Thelaira nigripes (FABRICIUS, 1794), male. 


\section{Genus Voria RoBINEAU-DESVOIDY, 1830}

\section{Voria ruralis (FALLÉN, 1810)}

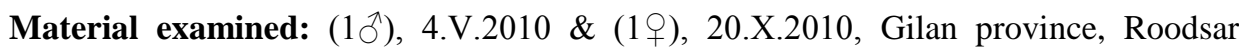

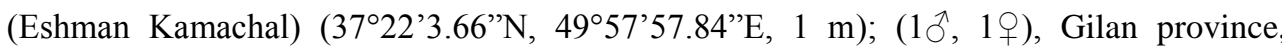
Roodsar (Ghazichak) (3645'57.54”N, 50¹9’35.22”E, 1803 m), 11.VI.2010; (1 q), Gilan province, Roodsar (Eshman Kamachal) (37²1'10.50”N, 49 57'56.16”E, 2 m), 30.X.2010, leg. M. KHEIRANDish; (19), Mazandaran province, Ghaznasara (36 $166^{\prime} 58.08^{\prime \prime} \mathrm{N}$, 52॰10’55.62’'E, 2013 m), 27.VII.2011, leg. L.A. NADIMI.

Distribution: Europe: Widespread in Europe (TSCHORSNIG et al. 2017). Asia: Transcaucasia, Central Asia, Middle East, Mongolia, Siberia, Russian Far East (HeRTING \& Dely-Draskovits 1993, ZiEGLER \& ShIMA 1996), Pakistan (O'HARA et al. 2009), Iran (PARCHAMI ARAGHI 1995).

Host range: Autographa gamma LINNAEUs; Spodoptera exigua (HÜBNER, 1808); Diachrysia chrysitis (LINNAEUS, 1758) (Lepidoptera: Noctuidae) (TSCHORSNIG \& HERTING 1994).

\section{DISCUSSION}

In the present study, the tribe Voriini comprises 7 genera and 8 species, among them three species are new records for the Iranian tachinid fauna. Periscepsia spathulata has a wide Western Palearctic distribution; the occurrence of this species in Transcaucasia and China was reported previously (HERTING \& DELY-DRASKOVITS 1993, TSCHORSNIG et al. 2017). Stomina caliendrata is widespread in the Western Palearctic and has an Asian distribution in Transcaucasia and Israel (HERTING \& DELY-DRASKOVITS 1993, TSCHORSNIG et al. 2017).

Another tribe - Dexiini - has two genera and two species. Estheria petiolata is a new record for the Iranian tachinid fauna. This species is widely distributed in the Western Palearctic and has been reported from Transcaucasia, Kazakhstan and Central Asia (HERTING \& DELY-DRASKOVITS 1993, TSCHORSNIG et al. 2017).

The present study increases the number of known Iranian Dexiinae species from 23 to 27. More intensive studies in northern Iran should be carried out: they would lead to a considerable increase in the number of known species from this area. 


\section{ACKNOWLEDGEMENTS}

We would like to express our cordial gratitude to Dr Joachim ZIEGLER (Museum of Natural History, Leibniz Institute, Berlin, Germany) and Dr Theo ZeEgERs (Eikenlaan, Soest, The Netherlands) for their kind assistance in confirming the identifications of the study materials. We also thank Dr Ahmad NADIMI and Dr Mohammad KHEIRANDISH for their assistance in collecting the materials.

\section{REFERENCES}

Cerretti P., Tschorsnig H-P. 2012. Three new species of Estheria Robineau-Desvoidy (Diptera: Tachinidae) from the Mediterranean, with a key to the European and Mediterranean species of the genus. Stuttgarter Beiträge zur Naturkunde A, Neue Serie 5: 271-286.

Gheibi M., Ostovan H., Kamali K. 2008. Fauna of parasitoid flies belonging to subfamilies Dexiinae and Phasiinae (Diptera: Tachinidae). [in:] SH. MANZARI (ed.) Proceedings of the $18^{\text {th }}$ Iranian Plant Protection Congress, 24-27 August 2008, University of Bu-Ali Sina, Hamedan, Iran. University of Bu-Ali Sina, Hamedan, 75.

Gheibi M., Ostovan H., Kamali K. 2009. A contribution to knowledge of the tachinid fly fauna of Fars province, Iran (Diptera: Tachinidae: Dexiinae and Phasiinae). Zoology in the Middle East 46(1): 69-74.

GRENIER S. 1988. Applied biological control with tachinid flies (Diptera, Tachinidae): a review. Anzeiger für Schädlingskunde, Pflanzenschutz, Umweltschutz 51(3): 49-56.

Herting B. 1984. Catalogue of Palearctic Tachinidae (Diptera). Stuttgarter Beiträge zur Naturkunde, Serie A (Biologie) 369: 1-228.

Herting B., Dely-Draskovits Á. 1993. Family Tachinidae. [in:] Á. Soós, L Papp. (eds.). Catalogue of Palaearctic Diptera. Volume 13, Anthomyiidae - Tachinidae. Akadémiai Kiadó. Budapest, 1309-458.

MESNIL L.P. 1944-1975. Die Fliegen der palaearktischen Region 10. 64g. Larvaevorinae (Tachininae). E. Schweizerbart'sche Verlagsbuchhandlung, Stuttgart.

MeSNIL L.P. 1980. Die Fliegen der palaearktischen Region 9. 64f. Dexiinae. E. Schweizerbart'sche Verlagsbuchhandlung, Stuttgart, 1-52.

O’HARA J.E. 2013. History of tachinid classification (Diptera, Tachinidae). ZooKeys 316: 1-34.

O'HARA J.E. 2014. World genera of the Tachinidae (Diptera) and their regional occurrence. Version 8.0. Internet: http://www.nadsdiptera.org/Tach/WorldTachs/Genera/Gentach_ver8.pdf (accessed 25.12.2016)

PARCHAMI ARAGHI M. 1995. Introduction of Voria ruralis (Dip.: Tachinidae), parasitoid of Autographa gamma (Lep.: Plusiidae) larva in Iran. [in:] Gh. RAJABI, A.M. SARAFrAZI, H. MirzaIAns (eds.). Proceedings of the $12^{\text {th }}$ Iranian Plant Protection Congress, 2-7 September 1995, Junior College of Agriculture, Karaj, Iran. University of Tehran, Karaj, 346. 
SAmet K., FArZaneh A., Barkhordari M. 1977. Preliminary list of Diptera (Tachinidae) of Iran, Journal of Entomological Society of Iran 4(1-2): 83-86.

Seyyedi Sahebari F., Khaghaninia S., Ziegler J. 2014. Faunistic study of tachinid flies of the subfamily Dexiinae (Diptera: Tachinidae) in northwestern Iran. Studia dipterologica 21(2): 243-256.

Stireman J.O., O'Hara J.E., Wood D.M. 2006. Tachinidae: Evolution, behavior, and ecology. Annual Review of Entomology 51: 525-555.

TsChORSNIG H.P. 1985. Taxonomie forstlich wichtiger Parasiten: Untersuchungen zur Struktur des männlichen Postabdomens der Raupenfliegen (Diptera, Tachinidae). Stuttgarter Beiträge zur Naturkunde, Serie A (Biologie) 383: 1-137.

Tschorsnig H.P., Herting B. 1994. Die Raupenfliegen (Diptera: Tachinidae) Mitteleuropas: Bestimmungstabellen und Angaben zur Verbreitung und Ökologie der einzelnen Arten. Stuttgarter Beiträge zur Naturkunde, Serie A (Biologie) 506: 1-170.

TSCHORSNIG H.P., Richter V.A. 1998. Family Tachinidae. [in:] L. PAPP, B. DARVAS (eds.) Contributions to a Manual of Palaearctic Diptera (with special reference to flies of economic importance). Higher Brachycera, Volume 3. Science Herald, Budapest, 691-827.

Tschorsnig H.P., Richter V.A., Cerretti P., ZeEgers T., Bergström C., VAnhara J., VAn de Weyer G., Bystrowsky C., RAPer C., Ziegler J., Hubenov Z. 2017. Fauna Europaea: Tachinidae. [in:] T. PAPE, P. Beuk (eds.). Fauna Europaea: Diptera, Brachycera. Database version 2.4. Internet: http://www.faunaeur.org (accessed 10.06.2017)

Wood D.M. 1987. Tachinidae. [in:] J.F. Mc Alpine, B.V Peterson., G.E. Shewell, H.J. Teskey, J.R. Vockeroth, D.M. Wood (eds.). Manual of Nearctic Diptera. Volume 2. Research Branch Agriculture Canada, Ottawa, 1193-1269.

Ziegler J., Shima H. 1996. Tachinid flies of the Ussuri area (Diptera, Tachinidae), Contributions to the knowledge of East Palaearctic insects, No. 5. Beiträge zur Entomologie 46: 379-478.

Ziegler J., Lutovinovas E., ZHANG Ch. 2016. The taxa of the Dinera carinifrons species complex (Diptera, Tachinidae), with the description of a new West Palaearctic subspecies and three lectotype designations. Studia dipterologica 21 (2): 249-275.

Received: 8 October 2017

Accepted: 21 January 2018 